\title{
Adaptive Robust Short-Term Planning of Electrical Distribution Systems Considering Siting and Sizing of Renewable Energy Based DG Units
}

\author{
Ozy Daniel Melgar-Dominguez ${ }^{\circledR}$, Mahdi Pourakbari-Kasmaei ${ }^{\circledR}$, Member, IEEE, \\ and José Roberto Sanches Mantovani, Member, IEEE
}

\begin{abstract}
Short-term planning is a decision-making process that aims at ensuring proper performance of electrical distribution systems (EDSs) within a short period of time. In recent years, this process has faced a significant challenge due to the integration of renewable energy based technologies. To handle such complicated planning problem most suitably, sophisticated algorithms are required. This paper proposes a mixed-integer linear programming model to find the optimal short-term plan of EDSs considering siting and sizing of capacitor banks and renewable energy sources, conductor replacement of overloaded circuits, and voltage regulators allocation. Besides considering the economic aspects, the environmental issues are also considered to promote a low carbon emission system. To address the uncertainties of electricity consumption and renewable energy output power, a two-stage robust optimization model is used, and to handle this model more efficiently, the column and constraint generation algorithm is applied. A 135-node distribution system is studied under different conditions to assess the performance of the proposed approach. Results show that the planning actions, for each case study, improve the efficiency of EDS and mitigate the pollutant emissions at the distribution level.
\end{abstract}

Index Terms-Adaptive robust model, environmental issues, renewable-based DG units, short-term planning problem.

\section{NOMENCLATURE}

\section{A. Sets and Indexes}

$\Omega^{c b} / C T \quad$ Set of CB capacities / conductor types.

$L / N \quad$ Set of circuits / nodes.

$T / Y \quad$ Set of time intervals / horizon planning (years).

$a, \dot{a} \quad$ Index of new and initial conductor type, respectively.

Manuscript received November 14, 2017; revised March 13, 2018; accepted April 17, 2018. Date of publication April 19, 2018; date of current version December 14, 2018. This work was supported in part by the Brazilian institutions CAPES, CNPq under Grant 305318/2016-0 and in part by the São Paulo Research Foundation-FAPESP under Grant 2015/21972-6. Paper no. TSTE01038-2017. (Corresponding author: Ozy Daniel Melgar-Dominguez.)

O. D. Melgar-Dominguez and J. R. S. Mantovani are with the Department of Electrical Engineering, São Paulo State University, Ilha Solteira 15385000, Brazil (e-mail: ozzy.damedo@gmail.com; mant@dee.feis.unesp.br).

M. Pourakbari-Kasmaei is with the Department of Electrical Engineering and Automation, Aalto University, Espoo 02150, Finland (e-mail: Mahdi.Pourakbari @ aalto.fi).

Color versions of one or more of the figures in this paper are available online at http://ieeexplore.ieee.org.

Digital Object Identifier 10.1109/TSTE.2018.2828778 $b$

$k / v$

$l, l^{\prime}$

$t / y$

B. Constants

$e^{p} \quad$ Pollutant emission coefficient.

$\hat{f}_{t}^{D}, \hat{f}_{t}^{G} \quad$ Expected demand and renewable output power factors at time $t$.

$f_{t}^{G^{w t}}, \bar{f}_{t}^{G^{w t}} \quad$ Lower and upper limits for WT output power factor $C^{p v}$ at time $t$.

$\underline{f}_{t}^{G^{p v}}, \bar{f}_{t}^{G^{p v}} \quad$ Lower and upper limits for PV output power factor at time $t$.

$\underline{f}_{t}^{D}, \bar{f}_{t}^{D}$

$\overline{P E}^{p}$

$\bar{I}_{a}$

IC

$l_{l}$ $N^{P V / W T}$

$P^{D}, Q_{m, y}^{D}$

$\bar{P}^{P V / W T}$

$Q_{b}^{e s p}$

$R, X, Z_{a}$

$R \%$

$P^{*}, Q^{*}, V^{*}$

$\bar{U}, \underline{U}$

$\zeta_{t, y}^{G}$
$\zeta_{b}^{c b^{f x / s w}}$
$\zeta_{\dot{a}, a}^{c r}$
$\zeta^{v r}$
$\zeta^{p v / w t}$
$\zeta_{y}^{p v / w t^{o p}}$
$\Delta$
$\underline{\Phi}_{y}^{S}, \bar{\Phi}_{y}^{S}$
$\Phi^{p v}, \Phi^{w t}$

Index of reactive power capacity of installed CB. Index of WT units / PV modules.

Index of circuits between nodes $m, n$ and $n, m$. Index of time intervals / planning years.

Lower and upper limits for electricity consumption factor at time $t$.

Maximum allowable pollutant emission.

Upper limit of the square of the current magnitude for cable $a$.

Investment cost limit.

Length of circuit $l$.

Maximum PV/WT DG units to be installed.

Active and reactive power demand at node $m$ and year $y$.

Active power capacity of PV module / WT unit.

Specified reactive power of CB with capacity $b$.

Resistance, reactance, and impedance of cable $a$.

Regulation \% of the VR to be installed.

Estimated operating point.

Upper and lower limits of the square of the voltage magnitude.

Energy cost at time interval $t$ of year $y$.

Installation costs for the CB type with capacity $b$.

Replacement cost of conductor $\dot{a}$ to $a$.

Installation cost of VR.

Installation costs of PV modules and WT units.

Operation and maintenance costs of installed PV modules and WT units at year $y$.

Weight to transform a year to time intervals.

Lower and upper substation power factors at year $y$.

Power factor for PV and WT DG units. 


\section{Continuous Variables}

$f_{t}^{D}, f_{t}^{G} \quad$ Demand and renewable generation power factor at time $t$.

$I_{l, a, t, y} \quad$ Square of current flow magnitude at circuit $l$, cable $a$, time $t$, year $y$.

$P, Q_{l, a, t, y}$ Active and reactive power flow at circuit $l$, cable $a$, time $t$, year $y$.

$P_{m, t, y}^{P V / W T} \quad$ PV/WT active power generation at node $m$, time $t$, year $y$.

$P^{S}, Q_{t, y}^{S} \quad$ Active and reactive power supplied by substation at time $t$, year $y$.

$Q_{m, t, y}^{C B} \quad$ Reactive power delivered by the CB at node $m$, time $t$, year $y$.

$Q_{m, t, y}^{P V / W T} \quad$ Reactive power supplied by PV/WT at node $m$, time $t$, year $y$.

$U, \hat{U}_{m, t, y} \quad$ Square of voltage magnitude and auxiliary variable to control $U$ by VR at node $m$, time $t$, year $y$.

\section{Binary and Integer Variables}

$C_{m, t, y} \quad$ Integer variable to define the $\mathrm{CB}$ modules at node $m$, time $t$, and year $y$.

$\bar{C}_{m} \quad$ Integer variable to define the maximum CB modules installed at node $m$.

$x_{l, a}^{c r}$

$x_{m, b}^{f x / s w}$ Binary variable to replace the conductor $a$ at circuit $l$.

Binary variables that define the capacity $b$ and $\mathrm{CB}$ type to be installed at node $m$ with power capacity $b$.

$x_{m, v / k}^{p v / w t}$

Binary variables for renewable DG units allocation at node $m$.

$x_{n}^{v r} \quad$ Binary variable for VR allocation at node $n$.

\section{INTRODUCTION}

D ISTRIBUTION companies (DISCOs) recently are facing multiple challenges to meet the needs and preferences of the consumers. These challenges are accentuated due to increase in demands that results in decreasing the quality and reliability of the supplied energy. In practice, DISCOs need to fulfill several objectives simultaneously. In this regard, this work investigates the strategies to ensure proper performance of the electrical distribution systems (EDSs) and thus guarantee the quality and reliability of the supplied energy via a robust optimization approach.

Traditionally, a classical strategy that DISCOs may be used to fulfill the network's immediate needs is through short-term planning actions such as siting, sizing, and replacements of some devices to provide effective control over the voltage magnitude, operational energy losses, reactive power flow, and power factor of the system. Among all, the voltage regulators (VRs) and capacitor banks (CBs) allocation, conductor replacement, and network reconfiguration are the most common actions in short-term planning [1]-[5]. However, with the emergence of renewable technologies, the performance of the EDS has been altered, and from the optimization perspective, the complexity of EDS has mainly increased due to the high level of uncertainties associated with these technologies. Therefore, this issue needs to be adequately addressed to avoid any adverse effect on the efficiency of the EDS [6]. This integration has been widely studied in multiples instances, especially photovoltaic (PV) and wind turbine (WT) technologies, to maximize the efficiency of an EDS [6]-[8].

Due to the importance of having an appropriate plan, combining several alternatives actions has received more attention. A planning scheme considering renewable and dispatchable distribution generation (DG) units and CBs was presented in [9] where a hybrid method based on tabu search and a genetic algorithm was used as the solution tool. In [10], a method for allocation of CBs with PV- and WT-based DG units was proposed, and a non-dominated sorting genetic algorithm was used to optimize these actions simultaneously. A stochastic methodology that maximizes the installed capacity of the renewable-based DG units while minimizing the energy losses and consumption of loads was proposed in [11]. An information-gap decision theory-based model that was proposed in [12] where the allocation of dispatchable DG units and circuit reinforcement were considered in an uncertain environment. Moreover, a short-term planning approach for conductor replacement of circuits, siting and sizing of wind-based DG units and $\mathrm{CBs}$, and protection devices of EDSs was proposed in [13] where a genetic algorithm was used to find the solution of this problem.

Recently, carbon emissions mitigation has become a quintessential challenge in the electricity sector, since this industry is responsible for a significant share of global greenhouse gas emissions [14]. As a result, the adoption of low carbon technologies related to renewable-based DG units has been promoted to attend the environmental targets [15]. However, in most of the works for the sake of simplicity, considering the environmental issues has been disregarded. In [16], an integrated planning methodology considering renewable-based DG units and demand response was proposed to improve the efficiency of the EDS, where the emissions were mitigated through of a monetary form by carbon taxation. In the same way, in [17], a strategic planning approach for finding the optimal allocation of renewable energy sources and reactive power support devices was proposed aiming at minimizing the distribution power losses, enhancing the voltage stability, and reducing the $\mathrm{CO}_{2}$ emission. In [18], a planning scheme to find the optimal site and size of renewable and dispatchable DG units using particle swarm optimization-based approach was proposed where different technologies based on PV, WT, and biomass were taken into account considering technical, economic, and environmental performance indices. An environmentally committed shortterm planning model considering PV-based DG units allocation was proposed in [19]. This planning model was based on a deterministic environment, minimizing the operational and investment costs while managing the emissions from the distribution side.

All in all, none of the works in literature has studied an uncertainty-based short-term planning model in the presence of several alternative planning actions. To the best of our knowledge, until now there exists no strategic short-term planning model to address the economic, operational, and environmental aspects of a distribution network via a two-stage robust formulation [20]. Therefore, the primary objective of this paper is to develop an integrated planning framework that takes advantage of the benefits of each investment alternative to max- 
imize the efficiency of an EDS by taking into account not only economic aspects but also the environmental issues to promote a low carbon emission system. This paper incorporates various actions such as siting and sizing of reactive support devices and renewable-based DG units, specifically PV and WT units, conductor replacement of overloaded circuits, and voltage control devices. This combinatorial optimization problem is inherently a mixed integer nonlinear programming (MINLP) problem that is approximated by a mixed integer linear programming (MILP) model. In order to approach the uncertainty in demand and renewable output power, an adaptive robust optimization problem, which is a three level optimization problem, is proposed. Thus, to solve this problem the column and constraint generation $(\mathrm{C} \& C G)$ algorithm was applied. To validate and show the effectiveness of the proposed approach, a 135-node distribution system was tested under different conditions. The robust planning framework was implemented in a mathematical programming language AMPL, and the commercial solver CPLEX was used to obtain its solution.

Therefore, the contribution of this work is threefold.

- Proposing an adaptive robust formulation for short-term planning problem of distribution systems; this formulation, besides improving the quality and reliability of energy provided to end-users, promotes a sustainable EDS with emissions abatement at the distribution level.

- Proposing an integrated short-term planning model that addresses the economic, operational, and environmental issues of an EDS; for which the uncertainty and variability of renewable-based DG units and consumers' demands are entirely taking into account.

- Obtaining a solver-friendly adaptive robust MILP model using appropriate linearization techniques; this way, using MILP solver guarantees to find the optimal global solution with high computational efficiency.

The rest of this paper is organized as follows. Section II presents the proposed adaptive robust model in detail. The solution framework is presented in Section III. In Section IV, assumptions, technical and economic data of test system, and case studies are presented. Section V contains the numerical results and discussions. Concluding remarks are presented in Section VI.

\section{PROBLEM ForMULATION}

This section describes the mathematical formulation of the proposed model that aims at obtaining the best short-term plan for EDSs via a flexible and multi-choice strategy by simultaneously determining the: a) optimal site, size, and type of CBs to be installed, b) the VR optimal location to control the voltage drop, c) the optimal conductors replacement of overloaded circuits through suggesting the respective new conductors, and d) optimal site and size of renewable-based DG units to be installed. The resulted model of this mathematical representation is inherently a MINLP model, that via appropriate linearization techniques, this model is recast to an approximated MILP model which is presented via a deterministic model (subsect.II-B). In order to consider the uncertainty of the renewable output power and demand consumption, this deterministic MILP model is extended to an adaptive robust model (subsect.II-C).
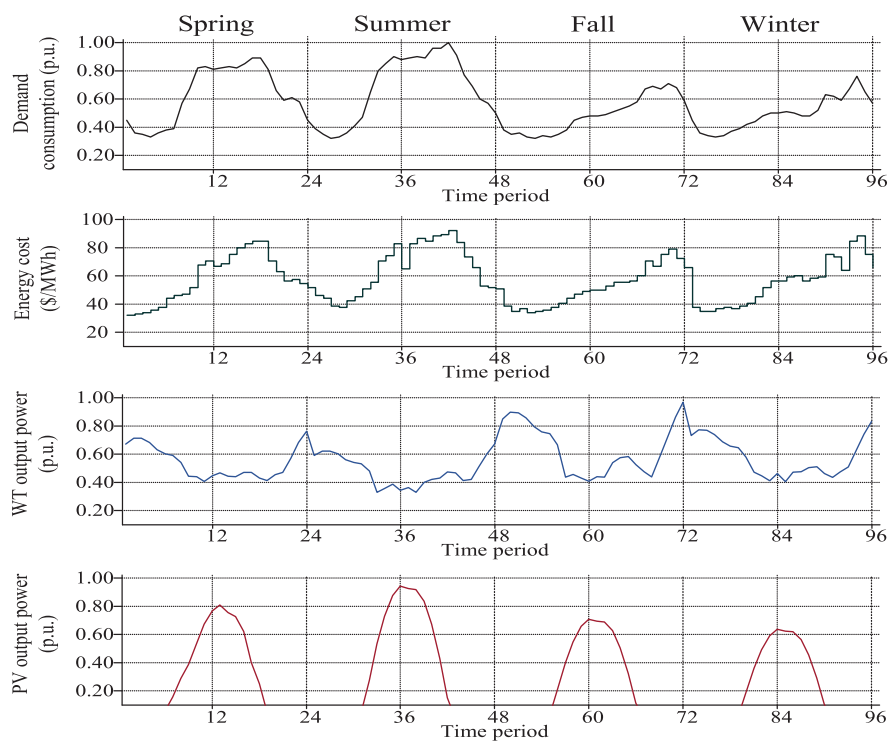

Fig. 1. Expected value of demand consumption, energy cost, WT and PV generation power.

\section{A. Demand and Renewable Energy Forecast Profiles}

Uncertainty is an inseparable part of demand, wind speed, and solar irradiation. Therefore, to consider such character in the proposed planning framework, this subsection describes the modeling in the presence of uncertainties arising from the renewable power generation and electricity consumption. It is worth mentioning that, the renewable output power can be created with weather forecast profiles, along with the horizon planning, and renewable production models [21], [22]. In this regard, for a proper analysis and to consider the influence of weather factor and seasonal characteristics in the renewable-based DG units planning, a year is represented with a time period $T$. This $T$ is constituted by time intervals $t \mathrm{~s}$, which could be divided in an hourly resolution $(T=8760 \mathrm{~h})$. However, considering such time-resolution results in an intractable problem. An appropriate representation can be properly defined through four blocks, as can be seen in Fig. 1, each block contains a typical seasonal day; therefore a year is composed by $96-t$ s. Each $t$ contains the correlation between demand consumption, energy cost, and electricity produced by renewable energy. In Fig. 1, for each $t$, the expected values of demand consumption, energy cost, and electricity produced by renewable energy are represented. In this approach, to simulate these expected profiles in demand consumption, historical demand forecast profiles are used. On the other hand, to simulate the expected renewable (PV and WT) output power, the online tool, "renewable.ninja", is used, while the historical data are obtained from [23] and [24]. It is noteworthy to mention that in the proposed approach, the average energy costs obtained from [25] are used.

Taking into account tropical countries such as Brazil, due to the temperature profile, two blocks of seasonal conditions are considered. In this regard, spring and summer are represented as hot weather conditions, while mild weather conditions constitute fall and winter. Considering these blocks of hot and mild weather, typical time intervals are reduced and are represented by its average $t$. In other words, the average $t$ represents time intervals with similar characteristics; thus, each $t$ contains data 
information related to demand, energy cost, and the output of renewable energy. This information is built considering an estimation along the planning horizon.

\section{B. Deterministic Formulation}

The proposed MILP model of the short-term planning problem with siting and sizing of renewable-based DG units considering multiple investment alternatives is presented as (1)-(26). This formulation defines an economic-environmental strategy to obtain the best short-term plan for an EDS. In the proposed model the following assumptions are taken into account.

- The planning framework is related to a centralized structure, for which all the technologies considered in the scope of this work, are owned and operated by the DISCO.

- Renewable-based DG units have the capability of connecting to a medium-voltage system, operating with the desired power factor, and controlling their power output by smart power inverters.

- The DISCO can define an investment limit for each alternative in order to obtain a suitable short-term plan.

The optimization model aims at minimizing: a) energy cost supplied by substation along the horizon planning (1st term), b) investment costs, in the initial year of the horizon planning, due to $\mathrm{CBs}$ allocation (2nd and 3rd terms), conductor replacement of overloaded circuits (4th term), WT generation allocation and its operation and maintenance cost (5th and 6th terms), siting and sizing of PV modules with respective operation and maintenance cost (7th and 8th terms), and VRs allocation (9th term). These investment alternatives are consequently represented in the optimization model, to maximize the efficiency of the EDS considering the technical, operational, and environmental aspects that must be satisfied to obtain a reliable and environmentally committed system.

$$
\begin{aligned}
& \min \sum_{t \in T} \sum_{y \in Y} \zeta_{t, y}^{G} \Delta P_{t, y}^{S}+\sum_{b \in \Omega^{c b}} \sum_{m \in N} \zeta_{b}^{c b^{f x}} x_{m, b}^{f x} \\
& +\sum_{b \in \Omega^{c b}} \sum_{m \in N} \zeta_{b}^{c b^{s w}} x_{m, b}^{s w}+\sum_{a \in C T} \sum_{l \in L} \zeta_{a}^{c t} l_{l} x_{l, a}^{c r}+\sum_{k=1}^{W T} \sum_{m \in N} \zeta^{w t} k x_{m, k}^{w t} \\
& +\sum_{y \in Y} \sum_{k=1}^{\overline{W T}} \sum_{m \in N} \zeta_{y}^{w t^{o p}} k x_{m, k}^{w t}+\sum_{v=1} \sum_{m \in N} \zeta^{p v} v x_{m, v}^{p v} \\
& \quad+\sum_{y \in Y} \sum_{v=1} \sum_{m \in N} \zeta_{y}^{p^{v o p}} v x_{m, v}^{p v}+\sum_{l \in L} \zeta^{v r} x_{l}^{v r}
\end{aligned}
$$

Subject to:

$$
\begin{aligned}
& F\left(x^{f x}, x^{s w}, x^{c r}, x^{v r}, x^{p v}, x^{w t}\right) \leq I C \\
& \sum_{l \in L} \sum_{a \in C T} P_{l, a, t, y}-\sum_{l^{\prime} \in L} \sum_{a \in C T}\left(P_{l^{\prime}, a, t, y}+R_{a} I_{l, a, t, y}\right) \\
& \quad+P_{t, y}^{S}+P_{m, t, y}^{P V}+P_{m, t, y}^{W T}=P_{m, y}^{D} f_{t}^{D} \\
& \sum_{l \in L} \sum_{a \in C T} Q_{l, a, t, y}-\sum_{l^{\prime} \in L} \sum_{a \in C T}\left(Q_{l^{\prime}, a, t, y}+X_{a} I_{l, a, t, y}\right) \\
& \quad+Q_{t, y}^{S}+Q_{m, t, y}^{P V}+Q_{m, t, y}^{W T}+Q_{m, t, y}^{C B}=Q_{m, y}^{D} f_{t}^{D}
\end{aligned}
$$

$$
\begin{aligned}
& U_{m, t, y}-\hat{U}_{n, t, y} \\
& =2 \sum_{a \in C T}\left[\left(R_{a} P_{l, a, t, y}+X_{a} Q_{l, a, t, y}\right)-\left(Z_{a} l_{l}\right)^{2} I_{l, a, t, y}\right] \\
& I_{l, a, t, y}=\left(V_{n, t, y}^{*}\right)^{-2}\left[\left(P_{l, t, y}^{*}\right)^{2}+2 P_{l, t, y}^{*}\left(P_{l, a, t, y}-P_{l, t, y}^{*}\right)\right. \\
& \left.+\left(Q_{l, t, y}^{*}\right)^{2}+2 Q_{l, t, y}^{*}\left(Q_{l, a, t, y}-Q_{l, t, y}^{*}\right)\right] \\
& \underline{U} \leq U_{m, t, y} \leq \bar{U} \\
& 0 \leq I_{l, a, t, y} \leq x_{l, a}^{c r} \bar{I}_{a} \\
& -\sum_{t \in T} P_{t, y}^{S} \tan \left(\cos ^{-1} \underline{\Phi}_{y}^{S}\right) \leq \sum_{t \in T} Q_{t, y}^{S} \\
& \sum_{t \in T} Q_{t, y}^{S} \leq \sum_{t \in T} P_{t, y}^{S} \tan \left(\cos ^{-1} \bar{\Phi}_{y}^{S}\right) \\
& \sum_{t \in T} e^{p} \Delta P_{t, y}^{S} \leq \overline{P E}^{p} \\
& Q_{m, t, y}^{C B}=C_{m, t, y} Q_{b}^{e s p} \\
& 0 \leq \bar{C}_{m} \leq \sum_{b \in \Omega^{c b}} b x_{m, b}^{f x}+\sum_{b \in \Omega^{c b}} b x_{m, b}^{s w} \\
& \bar{C}_{m} \leq C_{m, t, y}+\sum_{b \in \Omega^{c b}} b x_{m, b}^{s w} \\
& 0 \leq C_{m, t, y} \leq \bar{C}_{m} \\
& \sum_{b \in \Omega^{c b}} x_{m, b}^{f x}+\sum_{b \in \Omega^{c b}} x_{m, b}^{s w} \leq 1 \\
& \sum_{b \in \Omega^{c b}} x_{m, b}^{f x} \leq 1 ; \sum_{b \in \Omega^{c b}} x_{m, b}^{s w} \leq 1 \\
& (1-R \%)^{2} \hat{U}_{n, t, y} \leq U_{n, t, y} \leq(1+R \%)^{2} \hat{U}_{n, t, y} \\
& -(\bar{U}-\underline{U}) x_{n}^{v r} \leq U_{n, t, y}-\hat{U}_{n, t, y} \leq(\bar{U}-\underline{U}) x_{n}^{v r} \\
& P_{m, t, y}^{P V}=\sum_{v=1}^{\overline{P V}} v x_{m, v}^{p v} \bar{P}^{P V} f_{t}^{G^{p v}} \\
& \left|Q_{m, t, y}^{P V}\right| \leq P_{m, t, y}^{P V} \tan \left(\cos ^{-1}\left(\Phi^{p v}\right)\right) \\
& P_{m, t, y}^{W T}=\sum_{k=1}^{\overline{W T}} k x_{m, k}^{w t} \bar{P}^{W T} f_{t}^{G^{w t}} \\
& \left|Q_{m, t, y}^{W T}\right| \leq P_{m, t, y}^{W T} \tan \left(\cos ^{-1}\left(\Phi^{w t}\right)\right) \\
& \sum_{m \in N} \sum_{v=1}^{\overline{P V}} x_{m, v}^{p v} \leq N^{P V} ; \sum_{m \in N} \sum_{k=1}^{\overline{W T}} x_{m, k}^{w t} \leq N^{W T} \\
& \sum_{v=1}^{\overline{P V}} x_{m, v}^{p v} \leq 1 ; \sum_{k=1}^{\overline{W T}} x_{m, k}^{w t} \leq 1 \\
& \sum_{a \in C T} x_{l, a}^{c r}=1 \\
& x^{c r}, x^{f x}, x^{p v}, x^{s w}, x^{v r}, x^{w t} \in\{0,1\} \\
& \forall b \in \Omega^{c b} ; a \in C T ; l, l \in L ; m, n \in N ; t \in T ; y \in Y
\end{aligned}
$$


This optimization model is subject to: 1) investment limits, 2) steady state operating constraints, 3) EDS operational and environmental limits, and 4) operational constraints of each investment alternative, which are described as follow.

1) Investment Limits: Constraint (2) represents the investment limits that can be defined by the DISCO and contains the costs of: $\mathrm{CB}$ and VR allocation, conductor replacement, and siting and sizing renewable DG units.

2) Steady State Operation: The set of (3)-(6) represent the steady state operation of an EDS. The active and reactive power balance is shown in (3) and (4), respectively. The voltage magnitude is determined by (5), while (6) represents the linear approximation around an estimated operating point to calculate the square of the current magnitude.

3) Environmental and Operational Limits: The operational and environmental limits to be fulfilled in an EDS in order to supply an environmentally committed high-quality service, are considered in (7)-(10), where (7) and (8) represent the voltage limits and the current magnitude limits of circuits, respectively; and the annual substation power factor is controlled by (9); and the emissions from the substation side are controlled by the environmental sector via setting a cap to manage the annual emissions in (10).

4) Investment Alternative Constraints: The allocation of CBs is defined in (11)-(16), where the reactive power injection for each installed CB is formulated in (11), and the capacity of the CB (fixed or switchable) is determined by (12) (e.g. the product of $b x^{f x}$ and $b x^{s w}$ describes the CB capacity of the installed type); (13) and (14) are used to optimally choose the type of $\mathrm{CB}$ to be installed where $\bar{C}=C$, if fixed, and $C<\bar{C}$, if switchable; (15) guarantees that only one type of $\mathrm{CB}$ can be installed at node $m$; and (16) defines the capacity of fixed or switchable CB to be installed at node $m$.

The mathematical model of VRs is shown in (17) and (18). In these linear equations, it is assumed that the voltage magnitude can vary within a regulation range $(R \%)$, the tap position is considered to be continuous, and a VR can be allocated at the final node $n$. Variable $U$ represents the square of voltage magnitude, therefore (17) and (18) are related with the square of the regulation range.

The allocation of renewable-based DG units is defined by (19)-(24). For a PV technology, the available PV generation is described by (19), which depends on the renewable generation power and the modules to be installed, e.g., investment in PV modules with maximum power capacity $\bar{P}^{P V}$ are associated with the decision of the product of $v x^{p v}$. The reactive power injection can be adjusted by considering the active power generation of PV and the predefined power factor, (20). The WT allocation is defined by (21) and (22), in which the available WT generation is presented in (21). This equation depends on the generation power associated with the WT technology, the maximum power capacity, and the product $k x^{w t}$, which determines the units to be installed. The WT reactive power (22) is controlled with the power factor and the available active power generation. The maximum numbers of PV and WT plants to be installed in the EDS and the size of each plant are determined by (23) and (24), respectively. Constraint (25) imposes that only one conductor type is selected for each circuit. Finally, (26) represents the binary nature of the decision variables.

\section{Robust Formulation}

Robust optimization determines an optimal solution that by considering a predetermined uncertainty set is immune to all uncertainty realizations since within this set the realization of the worst scenario is considered [26]. The robust formulation tends to be a conservative method, to address this issue, two-stage robust optimization, known as adjustable or adaptive robust optimization, was proposed in [27]. In this formulation, the first stage determines a partial solution of the problem before the uncertainty realization. However, the decision of the second stage will be adjusted according to the information obtained from the first stage and the uncertainty realization. Compared with less conservative approaches such as probabilistic methods, robust optimization presents significant differences that can be highlighted as a) the robust formulation does not allow infeasible solutions by guaranteeing constraints satisfaction while probabilistic methods do not provide such guarantees; therefore, the robust optimal solution is immune to all uncertainty realization within the defined set; and b) unlike less conservative approaches, limited information such as mean or expected value, and lower and upper uncertainty limits can be sufficient to represent the uncertainty data in a robust optimization model [26], [27].

1) Uncertainty Characterization: The deterministic formulation presented in Section II-B requires accurate information related to electricity consumption and renewable output power (Fig. 1). However, prediction errors occur when the data is not exactly defined. Therefore, sub-optimal solutions to the shortterm planning problem considering renewable-based DG units allocation is obtained. To address such prediction errors, an uncertainty interval is considered. The upper and lower limits of this uncertainty interval are estimated with a certain confidence interval. To build this interval, it is considered that in the time $t$ there are $n^{o}$ values for both demand consumption $\left(f^{D}\right)$ and renewable output power $\left(f^{G}\right)$, as can be seen in (27).

$$
t \rightarrow\left\{\begin{array}{l}
f_{t}^{D(1)}, f_{t}^{D(2)}, \ldots, f_{t}^{D\left(n^{o}-1\right)}, f_{t}^{D\left(n^{o}\right)} \\
f_{t}^{G(1)}, f_{t}^{G(2)}, \ldots, f_{t}^{G\left(n^{o}-1\right)}, f_{t}^{G\left(n^{o}\right)}
\end{array}\right\}
$$

From these values, which can be obtained through historical data or statistical analysis, the mean values for $f^{D}$ and $f^{G}$ are calculated and are represented by $\hat{f}^{D}$ and $\hat{f}^{G}$, respectively. For practical purposes, a confidence interval of $95 \%$ is approximated with the normal probability distribution. These confidence intervals, (28) and (29), are defined by the mean values \pm the product between the critical value $Z_{\alpha / 2}$ and the standard error $\sigma / \sqrt{n^{\circ}}$, where $\sigma$ and $n^{o}$ are set to 0.3 and 30, respectively.

$$
\begin{aligned}
& \underline{f}_{t}^{D}=\hat{f}^{D}-Z_{\alpha / 2} \frac{\sigma}{\sqrt{n^{o}}} ; \bar{f}_{t}^{D}=\hat{f}^{D}+Z_{\alpha / 2} \frac{\sigma}{\sqrt{n^{o}}} \\
& \underline{f}_{t}^{G}=\hat{f}^{G}-Z_{\alpha / 2} \frac{\sigma}{\sqrt{n^{o}}} ; \bar{f}_{t}^{G}=\hat{f}^{G}+Z_{\alpha / 2} \frac{\sigma}{\sqrt{n^{o}}}
\end{aligned}
$$

For the sake of simplicity, it is assumed that all renewablebased DG units' locations are subject to the same conditions. Considering such information and the uncertainty characterization, the variability range of PV and WT generation power and demand consumption is limited by its respective uncertainty interval. To control the conservatism level, the uncertainty budget is defined and can be adjustable by the parameter $\Upsilon$, where the 
dimensions that can take the worst case can be managed [26].

$$
\begin{aligned}
& f_{t}^{G^{w t}} \in\left[\underline{f}_{t}^{G^{w t}}, \bar{f}_{t}^{G^{w t}}\right] ; \sum_{t \in T} \frac{\left|f_{t}^{G^{w t}}-\hat{f}_{t}^{G^{w t}}\right|}{\left(\bar{f}_{t}^{G^{w t}}-\underline{f}_{t}^{G^{w t}}\right)} \leq \Upsilon_{y}^{w t} \\
& f_{t}^{G^{p v}} \in\left[\underline{f}_{t}^{G^{p v}}, \bar{f}_{t}^{G^{p v}}\right] ; \sum_{t \in T} \frac{\left|f_{t}^{G^{p v}}-\hat{f}_{t}^{G^{p v}}\right|}{\left(\bar{f}_{t}^{G^{p v}}-\underline{f}_{t}^{G^{p v}}\right)} \leq \Upsilon_{y}^{p v} \\
& f_{t}^{D} \in\left[\underline{f}_{t}^{D}, \bar{f}_{t}^{D}\right] ; \quad \sum_{t \in T} \frac{\left|f_{t}^{D}-\hat{f}_{t}^{D}\right|}{\left(\bar{f}_{t}^{D}-\underline{f}_{t}^{D}\right)} \leq \Upsilon_{y}^{D}
\end{aligned}
$$

The uncertainty set is built for each time interval $t$. As can be seen from the terms on the left side of (30)-(32), these constraints enforce the WT/PV output power and the demand consumption to lay between the respective bound $[f, \bar{f}]$. The terms on the right side of (30)-(32) define the uncertainty budgets that bound the deviation between renewable generation and demand with their respective mean values $\left(\hat{f}_{t}^{G^{w t / p v}}, \hat{f}_{t}^{D}\right)$ divided by the distance of the uncertainty interval at interval $t$, numerator and denominator, respectively.

2) Adaptive Robust Optimization Model: The robust counterpart for the deterministic model is reformulated in (33)-(35), which comprises three levels of optimization problem that represents the proposed short-term planning model.

$$
\begin{aligned}
& \min _{x} \sum_{b \in \Omega^{c b}} \sum_{m \in N} \zeta_{b}^{c b^{f x}} x_{m, b}^{f x}+\sum_{b \in \Omega^{c b}} \sum_{m \in N} \zeta_{b}^{c b^{s w}} x_{m, b}^{s w} \\
& +\sum_{a \in C T} \sum_{l \in L} \zeta_{\dot{a}, a}^{c t} l_{l} x_{l, a}^{c r}+\sum_{k=1}^{\overline{W T}} \sum_{m \in N} \zeta^{w t} k x_{m, k}^{w t} \\
& +\sum_{y \in Y} \sum_{k=1}^{\overline{W T}} \sum_{m \in N} \zeta_{d}^{w t^{o p}} k x_{m, k}^{w t}+\sum_{v=1}^{\overline{P V}} \sum_{m \in N} \zeta^{p v} v x_{m, v}^{p v} \\
& +\sum_{y \in Y} \sum_{v=1}^{\overline{P V}} \sum_{m \in N} \zeta_{d}^{p v^{o p}} v x_{m, v}^{p v}+\sum_{l \in L} \zeta^{v r} x_{l}^{v r}+\psi^{O p} \\
& \text { s.t. : (2), (12)-(16), (23)-(26). } \\
& \psi^{O p}=\left\{\max _{f^{G p v}, f^{G t}, f^{D}} \delta^{o p}\right\} \\
& \text { s.t. : (30)-(32) } \\
& \delta^{o p}=\left\{\min _{P^{S}, Q^{S}, U, I, P, Q} \sum_{t \in T} \sum_{y \in Y} \zeta_{t, y}^{G} \Delta P_{t, y}^{S}\right\} \\
& \text { s.t. : (3)-(11), (17) }-(22)
\end{aligned}
$$

The upper level ((2), (12)-(16), (23)-(26) and (33)) is associated with the investment decisions constraints. The middle level ((30)-(32), and (34)) characterizing the uncertainty realization for the installed renewable-based DG units and the demand of the EDS. The lower level ((3)-(11), (17)-(22), and (35)) is corresponding to the reaction of the EDS considering the planning actions and uncertainty realization.

However, the proposed model can be solved appropriately using decomposition techniques. Therefore, the generic form of the proposed adaptive robust optimization problem is represented by (36)-(39) to illustrate the application of the decomposition algorithm, in the next section. In this generic form, decision variables $\left(x^{f x}, x^{s w}, x^{c r}, x^{v r}, x^{p v}, x^{w t}, C, \bar{C}\right)$ of the first stage are represented by $x$ in the set $X$. The variables of the second stage $\left(P^{S}, Q^{S}, U, \hat{U}, I, P, Q, P^{P V}, Q^{P V}, P^{W T}, Q^{W T}\right)$ are represented by $z$ in the set $\Omega(f, x)$ and the uncertainty variables are represented by $f$ in the uncertainty set $F$.

$$
\begin{aligned}
& \min _{x \in X} c_{1}^{T} x+\psi^{O p} \\
& \text { s.t. : } c_{1}^{T} x \leq \Pi ; x \in\{0,1\} \\
& \psi^{O p}=\left\{\max _{f \in F} \delta^{o p}\right\} \\
& \delta^{o p}=\left\{\min _{z \in \Omega\left(f^{*}, x^{*}\right)} c_{2}^{T} z\right\}
\end{aligned}
$$

where, the upper level is represented by (36) and (37), the middle level by (38) and the lower level by (39). In the lower level problem, $\Omega\left(f^{*}, x^{*}\right)=\left\{G\left(x^{*}, f^{*}, z\right)=b_{1} ; H\left(x^{*}, f^{*}, z\right) \leq b_{2}\right\}$ which contains the equality and inequality constraints ensures the feasibility of the operating decision variables $z$ (steady state operation, EDS operational limits, and operational constraints of each planning alternative). It is worth noting that, $G\left(x^{*}, f^{*}, z\right)$ and $H\left(x^{*}, f^{*}, z\right)$ are parametrized by the solution obtained from the upper and middle level $\left(x^{*}=x\right.$ and $\left.f^{*}=f\right)$, and the parameters without uncertainty are represented by $c_{1}, c_{2}, b_{1}$, and $b_{2}$.

\section{SOLUTION FRAMEWORK}

The proposed tri-level (min-max-min) adaptive robust optimization model (36)-(39), which represents the short-term planning problem considering siting and sizing of renewablebased DG units, is a very complicated problem to be solved using classical optimization techniques. An approximated single-level model might be a way to solve this problem, however, an intractable problem may result. Due to the structure of the proposed problem, it cannot be solved directly using commercial solvers. In this regard, decomposition algorithms (e.g., Benders Decomposition) can be considered as a remedy to address such difficulties and to obtain a tractable problem.

In [28], the Column-and-Constraint Generation (C\&CG) as a different cutting plane strategy to solve two-stage robust optimization problems was presented. This algorithm for a determined uncertainty realization dynamically generates constraints with recourse variables in the primal space. Compared with the classical Benders, the $C \& C G$ creates primal cuts that are usually more powerful than the dual cuts used in Benders, and therefore, the C\&CG needs fewer iterations to converge. To guarantee the convergence of this algorithm, the exchange of information between the master problem (MP) and the sub-problem (SP) must be exact. In other words, the optimal global solution must be guaranteed for each problem. Consequently, this decomposition algorithm is used to solve the short-term planning problem proposed in this work.

\section{A. Hierarchical Structure of the Problem}

To solve the proposed model by using the $\mathrm{C} \& \mathrm{CG}$ algorithm, the reformulation of (36)-(39) in a hierarchical framework of an MP and SP scheme is necessary. To apply this decomposition 
algorithm, the MP is defined as (40)-(45).

$$
\begin{aligned}
& \min _{x \in X} c_{1}^{T} x+\eta \\
& \text { s.t. : } c_{1}^{T} x \leq \Pi \\
& x \in\{0,1\} \\
& \eta \geq c_{2}^{T} z_{o} \\
& G\left(x, f_{o}^{*}, z_{o}\right)=b_{1} \\
& H\left(x, f_{o}^{*}, z_{o}\right) \leq b_{2} \quad \forall o \in O
\end{aligned}
$$

This MP is a relaxation of the presented robust problem, where the variables $z$ are dependent on the realization $o$ in the set $O$ while the auxiliary variable $\eta$ is added; the uncertainty parameter $f$ is fixed to the $f_{o}^{*}$ (obtained from the SP), and the solution of the MP problem defines the appropriate investment alternatives $x$, and this information is fixed in the SP as $x^{*}$. The SP associated with this problem is defined by (46)-(49).

$$
\begin{aligned}
& \max _{f \in F} \delta^{o p} \\
& \text { s.t. : } \delta^{o p}=\left\{\min _{z \in \Omega(f, x)} c_{2}^{T} z\right\} \\
& G\left(x^{*}, f, z\right)=b_{1} \\
& H\left(x^{*}, f, z\right) \leq b_{2}
\end{aligned}
$$

This SP is a bi-level linear optimization problem, and in this work, by using the KKT optimality conditions [29], is recast to an equivalent MILP single-level model with complementary constraints (50)-(55).

$$
\begin{aligned}
& \max _{f, z} c_{2}^{T} z \\
& \text { s.t. : } G\left(x^{*}, f, z\right)=b_{1} \\
& f \in[\underline{f}, \bar{f}] ; f \in \Upsilon \\
& \frac{\partial}{\partial z}\left(c_{2}^{T} z+\lambda H\left(x^{*}, f, z\right)+\gamma G\left(x^{*}, f, z\right)\right)=0 \\
& 0 \leq b_{2}-H\left(x^{*}, f, z\right) \leq(1-w) \bar{M} \\
& 0 \leq \lambda \leq w \bar{M}
\end{aligned}
$$

where $\lambda$ and $\gamma$ represent the dual variables corresponding to the set of equations $H\left(x^{*}, f, z\right), G\left(x^{*}, f, z\right)$, respectively; (52) represents the uncertainty range and budget of the variables $f$; (53) is the differentiating of the lagrangian of the lower level problem; and (54) and (55) are the linearized expressions of the complementary conditions $\left[H\left(x^{*}, f, z\right)-b_{2} \leq 0, \lambda \geq 0\right.$, and $\left.\left.\lambda\left(b_{2}-H\left(x^{*}, f, z\right)\right)=0\right)\right]$, using the vector of binary variables $w$ and big $-\bar{M}$ method.

\section{B. The $C \& C G$ Algorithm}

The overall procedure of applying the C\&CG algorithm is summarized as follows:

Initial step: a) Solution of the MP defines the Lower Bound (LB) as $\mathrm{LB}=\eta^{*}$ while the Upper Bound (UB) is defined by the solution of the SP as UB = $c_{2}^{T} z^{*}$. Initially, set $\mathrm{LB}=-\infty$, and $\mathrm{UB}=\infty$.

b) Define the tolerance $t o l$ and the set $O \in \emptyset$. c) Denote $i$ as the suffix of algorithm iteration, initially set this value to 1 and define $O \cup i$.

d) Define the initial value of $f_{i} \in[f, \bar{f}]$ and $f_{o}^{*}=$ $f_{i} \forall o \in O$.

MP step: Solve the $\operatorname{MP}^{i}\left(f_{o}^{*}\right)$; with the solution $(x, \eta)$, update the $\mathrm{LB}=\eta$, and send $x^{*}=x$ to SP.

SP step: a) Solve $\mathrm{SP}^{i}\left(x^{*}\right)$, denote the solution $z, f$.

b) Update the $\mathrm{UB}=\min \left\{\mathrm{UB}, c_{2}^{T} z\right\}$.

Final step: If $($ gap $=(\mathrm{UB}-\mathrm{LB}) / \mathrm{LB} * 100 \%) \leq t o l$ then end, otherwise update $i=i+1, O \cup i$, and $f_{i}^{*}=f_{i-1}$ and go to MP step.

To solve the proposed short-term planning model, for the initial step the MP considers (43)-(45); in this regard, possible values of uncertain data (values within uncertainty interval) are the initial information used for the C\&CG algorithm, and for the next iterations, the solution of the SP is utilized. This procedure reduces the number of iterations in the C\&CG algorithm, since it starts with a feasible point, and thus the solution of the aforementioned problem can be obtained with higher computational efficiency.

\section{Solution Scheme for the Short-Term Planning Problem}

To solve the proposed adaptive short-term planning problem, a solution scheme should be duly represented. This solution scheme is divided into three successive stages and summarized in the Fig. 2. The first stage represents the estimation process, the second stage contains the robust optimization problem, and the third stage describes the planning actions to be carried out to maximize the efficiency of an EDS. These stages are explained in details as follow.

1) Estimation process: the proposed approach requires appropriate data information such as operating point $\left(V^{*}\right.$, $P^{*}$, and $Q^{*}$, and forecasted values of demand consumption and renewable energy. The initial operating point is obtained from a conventionally optimal power flow. This initial operating point and the forecasted values are sent to the deterministic linear programming (LP) model, where the integrality of all decision variables of the MILP model presented in II-B is relaxed. The solution obtained from the LP is used to update the operating point. Besides, in this stage, the uncertainty interval is estimated using the procedure presented in the Section II-C1. Finally, the updated estimated operating point and the uncertainty interval are sent to the second stage.

2) Robust optimization problem: this stage contains the adaptive robust optimization problem in a hierarchical environment. The C\&CG algorithm presented previously is used to solve this problem. It is worth mentioning that, in each iteration, the uncertainty realization is determined and the operating point is updated to improve the quality of the approximated model.

3) Planning actions: finally, this stage presents the planning actions to be carried out to improve the efficiency of the EDS as the best economic-environmentally strategy.

\section{CASE Studies}

The adaptive robust formulation described in Section II-C is tested on a 135-node distribution system presented in [5]. This 


\section{ESTIMATION PROCESS}

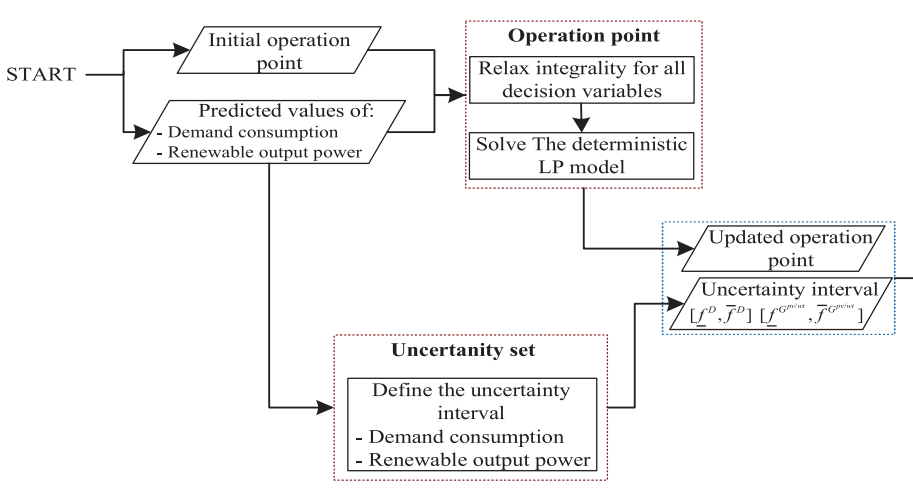

Fig. 2. Flowchart of the proposed solution framework.

section presents the technical and economic information of each investment alternative, assumptions, and case studies to validate the proposed model.

\section{A. Technical and Economic Information}

Test system information: The 135-node distribution system, illustrated in Fig. 4, contains 1 substation, 134 nodes (with 100 load nodes), and 133 circuits with different conductor types (47 with AA4 type, 43 with AA1 type, 23 with AA3 type, and 20 with AA2 type), [5]. The total conventional demand is $6.499 \mathrm{MW}$ and 2.769 MVAr. The nominal medium voltage level is $13.8 \mathrm{kV}$, while the lower and upper voltage limits are 0.95 and 1.05 p.u., respectively. The annual substation power factor can vary between 0.95 to 1.0 , and the reactive power depends on the power factor and the delivered active power.

$C B, V R$, and conductor replacement information: The regulation range and installation cost of VRs are assumed to be $10 \%$ and $\$ 21,350$, respectively; fixed and switchable CBs are considered where each CB type has its reactive power capacity and installation cost; six different conductor types with technical and economic characteristics are taking into account while to replace a specific conductor by new conductor a cost is associated. The technical and economic information about CBs and conductor replacement can be found in [5].

Renewable-based DG units information: PV and WT are the renewable generation technologies considered in this planning problem. The generation capacity of each WT unit and PV module is $100 \mathrm{~kW}$ and $20 \mathrm{~kW}$ considering power factors of 0.99 and 0.98 , respectively. The maximum number of WT units and PV modules that can be installed at node $m$ is set to 5 and 20, respectively. Investment costs of $\$ 100 \mathrm{k}$ and $\$ 20 \mathrm{k}$, and operation and maintenance costs of $\$ 150$ and $\$ 375$ are considered for each WT unit and PV module. The operating and maintenance costs are increased each year by $5 \%$. The technical and economic information was derived from [21].

\section{B. Assumptions and Cases}

In order to make a proper analysis and to validate the proposed approach, the main assumptions are summarized as follows: a) A planning horizon of 3 years is considered, b) the annual demand

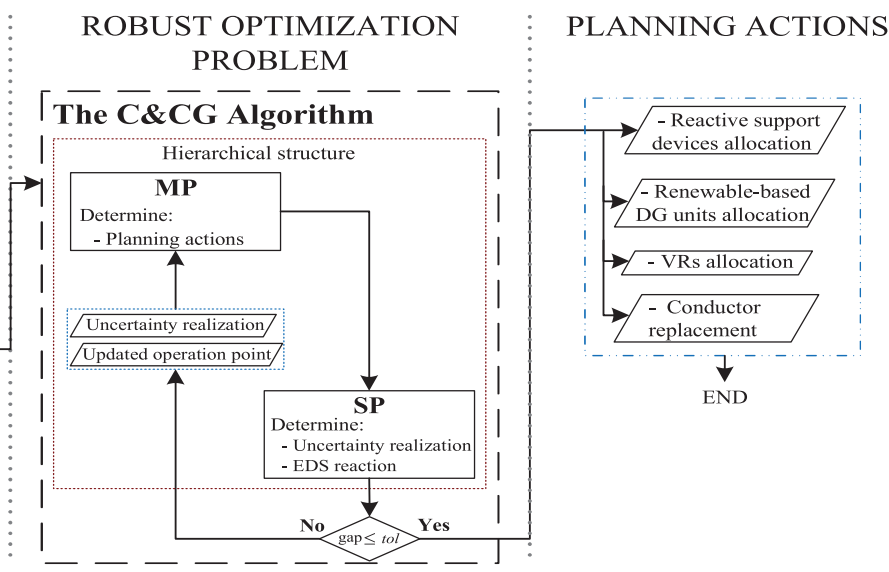

and the annual energy cost increases are $10 \%$ and 5\%, respectively; c) investment cost of the renewable-based DG units is limited to $\$ 800 \mathrm{k}$ per each technology (PV and WT); d) EDS reinforcement in VRs and CBs allocation, and conductor replacement are limited to $\$ 20 \mathrm{k}, \$ 42.7 \mathrm{k}$, and $\$ 50 \mathrm{k}$, respectively; e) the annual emission limit is $85 \mathrm{kTon}$ considering an intensity coefficient emission of $2.17 \mathrm{~kg} \mathrm{CO}_{2} / \mathrm{kWh}$ [30]; f) the robustness parameter $(\Upsilon)$ is set to 0.70 ; and $g$ ) the optimality gap is set to $0.5 \%$.

To validate the proposed methodology, case studies are carried out under two different conditions. For the first condition, namely moderate-resolution condition, demand consumption and renewable output power is represented via $12-t \mathrm{~s}$, which is an approximation of the second condition, namely high-resolution condition, with a higher time resolution of 96- $t \mathrm{~s}$ (see Fig. 1). We use the second condition to show the applicability and potential of the proposed approach for a higher time-resolution.

1) Moderate-Resolution Condition: Under this condition, four different cases are studied to validate the proposed approach. It is worth mentioning that before planning, an analysis via a conventional power flow is done to determine the initial conditions of the 135-node distribution system.

- Case I: Considering optimal allocation of CBs and VRs, and conductor replacement.

- Case II: Considering optimal allocation of CBs, VRs, and renewable-based DG units.

- Case III: Considering all the investment alternatives such as: CBs and VRs allocation, conductor replacement, and renewable-based DG units allocation.

- Case IV: Considering the same decisions variables of Case III via the deterministic model. In this case, expected values of the renewable generation and demand are used.

2) High-Resolution Condition: To demonstrate the robustness and applicability of the proposed planning framework, the aforementioned cases I-III are analyzed under a high-resolution condition with an annual representation of 96- $t \mathrm{~s}$.

\section{Simulation Results And Discussion}

The mathematical models and the solution framework presented were implemented in the mathematical language AMPL, and the commercial solver CPLEX was used to solve the robust 
optimization problem. A computer with an Intel Xeon E5-2650 $\mathrm{v} 4$ processor was used for the simulations. In this section, numerical results of the 135-node distribution system, under different conditions, are presented and duly discussed. To differentiate the moderate- and high-resolution conditions, case studies are represented by extensions .m (e.g., Case I.m, Case II.m, etc.) and .h (e.g., Case I.h, Case II.h, etc.), respectively.

\section{A. Moderate-Resolution Condition}

Performance of the proposed approach is validated under moderate-resolution conditions considering an approximated annual division of 12- $t \mathrm{~s}$. The maximum solution time found under this condition are 3 hours and 25 minutes. For comparative purpose, the initial condition of the 135-node distribution system was obtained via a conventional power flow for the first year, where the uncertainty of demand consumption is disregard. This solution evidences an initially violated 135-node distribution system in technical aspects. The voltage profile corresponds to the interval with the maximum loading of the first year, is depicted in Fig. 3 in red. As can be seen, the lower voltage limit has not met. Under this circumstances, the system power factor is 0.918 , and the substation energy cost is $\$ 2130.304 \mathrm{k}$ with 77.590 kTon of emissions.

1) Case I.m: This case aims at evaluating the proposed planning scheme considering the uncertainty of electricity consumption in the absence of renewable-based DG units allocation. The obtained solution reveals the investment alternatives such as a) allocation of a switchable $\mathrm{CB}$ at node 95 with a total cost of $\$ 10.15 \mathrm{k}$, b) allocation a VR device at node 8 with $\$ 21.35 \mathrm{k}$ investment cost, and c) replacement of conductors of 16 circuits by new conductors with spending $\$ 48.00 \mathrm{k}$.

It is worth mentioning that, due to the limited financial resources, the planning scheme obtained for this case could not address the environmental targets, (see Table I). Therefore, to get a feasible solution, the emission limit was relaxed. The emission limits in the second and third year of planning were exceeded approximately $0.11 \%$ and $9.38 \%$, respectively, considering an emission limit of $85 \mathrm{kTon}$.

2) Case II.m: The results obtained for this case reveals a total investment cost of $\$ 1106.19 \mathrm{k}$ that can be summarized as follows: a) optimal allocation of a fixed $\mathrm{CB}$ at node 107 with the investment cost of $\$ 8.075 \mathrm{k}, \mathrm{b}$ ) allocating a VR device at node 9 with the cost of $\$ 21.35 \mathrm{k}$, and c) siting and sizing of renewable DG units with total investment cost of $\$ 1076.77 \mathrm{k}$.

This planning scheme demonstrates that by allocating the renewable-based DG units, environmental limits can be met (see Table I). Results compared to the initial condition and the first case show significant reductions in emissions. Comparing with the initial condition, about $15.09 \%$ reduction resulted, while comparing with each planning year in Case I.m, about $14.6 \%, 13.11 \%$, and $11.68 \%$ of emission reduction have been obtained.

3) Case III.m: This case, by considering all the investment alternatives, aims at showing the potential and effectiveness of the proposed model in solving such complicated problems. The obtained results are summarized as follows: a) siting and sizing of a fixed CB at node 103 with $\$ 6.55 \mathrm{k}$ investment cost, b) allocating a VR device at node 63 with $\$ 21.35 \mathrm{k}$ installation cost, c) replacing conductors of 11 circuits by new ones with a total cost of $\$ 45.80 \mathrm{k}$, and d) siting and sizing of renewablebased DG units with a total investment cost of $\$ 994.882 \mathrm{k}$.

In this case, all the technical, operational and environmental aspects were satisfied. Considering the possibility of conductor replacement, the investment costs in renewable-based DG units reduced by $7 \%$ comparing with Case II.m, while the substation energy cost decreased by more than $1.15 \%$.

4) Case IV.m: In this case, the demand and generation profiles were set to the expected forecast profile, and these values were used to solve the deterministic model (Section II-B). The solution of this case reveals a total investment cost of $\$ 1049.502 \mathrm{k}$; this cost corresponds to the alternatives such as a) allocation of three fixed CBs with investment cost of $\$ 16.45 \mathrm{k}$, b) replacement of the conductors of 9 circuits with a cost of $\$ 38.17 \mathrm{k}$, and c) placement and sizing of renewable-based DG units with total cost of $\$ 994.882 \mathrm{k}$.

This case shows that the total emission from the substation side per year are $65.96 \mathrm{kTon}, 73.82 \mathrm{kTon}$, and 82.49 kTon, and the overall cost related to the substation supplied energy per year are $\$ 1851.970 \mathrm{k}, \$ 2174.361 \mathrm{k}$, and $\$ 2549.558 \mathrm{k}$, respectively. The total cost related to the substation supplied energy throughout the planning horizon is $\$ 6575.889 \mathrm{k}$, which is about $4.75 \%$ lower than the solution obtained in Case III.m.

5) Validation of Robust Solution of Case III.m: To validate the obtained solution for Case III.m, the set of planning action is fixed and a random profile related with the renewable output power and demand consumption, and the values between the uncertainty intervals are used to solve the OPF problem.

The solution of this OPF shows a feasible solution in which the emissions from the substation side for the planning years are 67.05 kTon, 74.94 kTon, and 83.67 kTon, corresponding with the total cost of substation supplied energy of $\$ 1880.975 \mathrm{k}$, $\$ 2206.372 \mathrm{k}$, and $\$ 2585.416 \mathrm{k}$, respectively. This planning scheme, compared to the initial conditions, demonstrates that the emissions and substation energy costs were decreased by $12.45 \%$ and $11.70 \%$, respectively. On the other hand, comparing these values with Case IV.m reveals that the emissions were increased by $1.58 \%, 1.47 \%$, and $1.40 \%$, for each planning year, corresponding with $1.54 \%, 1.45 \%$, and $1.39 \%$ increase in the substation energy costs. Results show that to address the technical, operational, and environmental issues, considering variations in the uncertainty of data, and the amount of energy provided by the substation must be higher than in the deterministic case, as can be seen in Table I for Case III.m, the energy cost of the substation was around $\$ 6900 \mathrm{k}$.

The solutions found by the robust and deterministic models present similar plans. However, the differences can be highlighted as a) the robust case proposes the replacement of conductors of two circuits that were not proposed in the deterministic case, b) the deterministic solution proposes the installation of 2 fixed CBs that were not installed in the robust solution, and c) in Case III.m, a VR was allocated. In order to meet the environmental targets, both models determine similar investment costs related to renewable-based DG units. The main difference is that the robust model avoids voltage magnitude violations due to the variability of uncertainty data. This fact can be seen in Fig. 3 where it shows a substantial improvement in the system voltage profile compared with the initial EDS state. This figure 


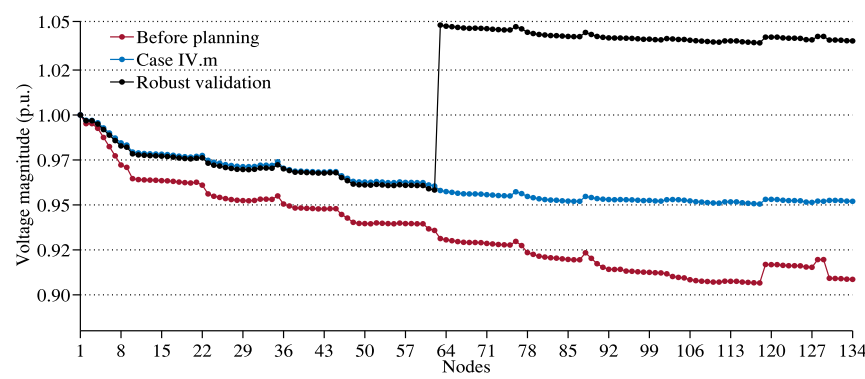

Fig. 3. Voltage profiles of 135-nodes system under peak loading time interval, for initial condition, deterministic Case IV.m, and robust validation of Case III.m.

contains three voltage profiles corresponding to before-planning (red line), robust validation (black line), and Case IV.m (blue line), under the peak loading interval. It is worth noting that, considering the solution of Case IV.m, variation in demand and renewable generation data may cause violations in the voltage profile.

\section{B. High-Resolution Condition}

In this subsection, the proposed approach is validated under a higher time-resolution. Under this condition, the maximum time solution found was about 116 hours. The obtained short-term plan for each case is summarized as follows.

1) Case I.h: The solution obtained for this case shows a total investment cost of $\$ 77.85 \mathrm{k}$. This investment cost is corresponding to the following actions: a) siting and sizing of a fixed $\mathrm{CB}$ at node 107 with investment cost of $\$ 7.50 \mathrm{k}$; b) allocating a VR device at node 9 with investment cost of $\$ 21.35 \mathrm{k}$; and c) replacing the conductors of 16 circuits by new conductors with investment cost of $\$ 49.00 \mathrm{k}$. The solution of this case proposes similar planning actions as Case I.m. However, some differences can be highlighted as a) Case I.m proposes a switchable $\mathrm{CB}$, meanwhile Case I.h proposes a fixed $\mathrm{CB}$; b) the total investment cost of Case I.m was $\$ 79.50 \mathrm{k}$, which represents $2 \%$ higher costs than Case I.h; c) the cost of energy supplied by the substation has a relative difference of $0.94 \%$ between Cases I.m and I.h (see Table I); and d) comparing the proposed conductor replacement between both cases, two circuits with different conductor types have been determined for Case I.h. This planning scheme is shown in Fig. 4 and Table II, where each planning alternative is presented in detail.

2) Case II.h: The short-term plan found in this case shows a total investment cost of $\$ 1058.13 \mathrm{k}$. This investment cost is related to the following actions: a) siting and sizing of a fixed and a switchable CB at nodes 107 and 90 with investment cost of $\$ 5.15 \mathrm{k}$ and $\$ 10.15 \mathrm{k}$, respectively, b) allocating a VR device at node 8 with investment cost of $\$ 21.35 \mathrm{k}$, and c) siting and sizing of renewable-based DG units with total investment cost (including operation and maintenance cost) of $\$ 1015.34 \mathrm{k}$. This set of planning actions is shown in Fig. 4, where the location and capacity for each planning action are duly identified.

The obtained solution for Case II.h proposes a similar investment plan as Case II.m. Nevertheless, these investment plans present some differences that can be highlighted as a) the total cost found for Case II.h, including investment cost and cost of energy supplied by substation (see Table I), was $\$ 8164.00 \mathrm{k}$
TABLE I

OPTIMAL UB AND LB VALUES, GAPS AND EMISSIONS FOR MODERATE- AND HIGH-RESOLUTION

\begin{tabular}{l|c|c|c|c|c|c|c}
\hline Case & Iteration & UB & LB & gap & \multicolumn{2}{|c|}{ Emissions } & (kTon) \\
\cline { 5 - 8 } & & $\left(10^{3} \$\right)$ & $\left(10^{3} \$\right)$ & $(\%)$ & $y_{1}$ & $y_{2}$ & $y_{3}$ \\
\hline I.m & 1 & 7868.31 & 7531.05 & 4.48 & 77.28 & 85.20 & 93.93 \\
& 2 & 7862.49 & 7853.61 & 0.11 & 77.16 & 85.09 & 93.80 \\
\hline II.m & 1 & 6997.68 & 6586.65 & 6.24 & 65.95 & 73.92 & 82.74 \\
& 2 & 6986.64 & 6976.50 & 0.15 & 65.88 & 73.93 & 82.84 \\
\hline III.m & 1 & 6961.86 & 6572.97 & 5.91 & 65.97 & 73.81 & 82.45 \\
& 2 & 6903.75 & 6901.38 & 0.03 & 65.99 & 73.84 & 82.54 \\
\hline I.h & 1 & 7793.88 & 7435.62 & 4.82 & 76.12 & 84.80 & 92.30 \\
& 2 & 7788.18 & 7784.70 & 0.04 & 76.60 & 85.03 & 92.80 \\
\hline II.h & 1 & 7147.38 & 6785.47 & 5.33 & 65.93 & 73.50 & 81.95 \\
& 2 & 7105.87 & 7095.27 & 0.15 & 65.95 & 73.56 & 82.10 \\
\hline III.h & 1 & 7060.33 & 6684.39 & 5.62 & 65.42 & 72.83 & 81.09 \\
& 2 & 7017.77 & 7013.87 & 0.05 & 65.85 & 73.31 & 81.66 \\
\hline
\end{tabular}

while the total cost for Case II.m was $\$ 8092.83$. The solutions have a relative difference of $0.81 \%$; b) in Case II.h two CBs are allocated (fixed and switchable) while in Case II.m only a fixed CB was allocated; c) investment cost in renewable-based DG units in Case II.h was $\$ 61.43 \mathrm{k}$ less than such investment cost in Case II.m. As can be seen from Fig. 4, the total installed capacity in Case II.m was $1060 \mathrm{~kW}$ while in Case II.h it was $1000 \mathrm{~kW}$; and d) for Case II.h the model obtains a higher cost of supplied energy by the substation than such expenses in Case II.m, approximately $1.68 \%$ higher (see Table I).

3) Case III.h: Similar to Case III.m, all the investment alternatives are taken into account. This case aims to show the potential and robustness of the proposed approach considering an annual representation of 96- $t \mathrm{~s}$. The obtained solution shows a set of planning actions to maximize the efficiency of the 135-node distribution system and to promote a low carbon emissions system. This set is summarized as follows: a) allocating two fixed CBs at nodes 90 and 103 with investment cost of $\$ 10.10 \mathrm{k}$; b) allocating a VR at node 63 with investment cost of $\$ 21.35 \mathrm{k} ; \mathrm{c}$ ) replacing conductors of 11 circuits with total cost of $\$ 44.22 \mathrm{k}$; and d) siting and sizing of DG units based on WT and PV with a total cost of $\$ 913.45 \mathrm{k}$ including operation and maintenance costs.

In this case, the total cost was $\$ 8023 \mathrm{k}$, considering the investment cost, $\$ 1009.13 \mathrm{k}$, and the cost of the supplied energy by the substation, $\$ 7013.87 \mathrm{k}$, see Table I. On the other hand, the total cost in Case I.m was $\$ 7969.96 \mathrm{k}$, comparing these values, a relative difference of $0.66 \%$ exists. However, similar short-term plans with some differences have been determined for cases III.m and III.h. These differences can be highlighted as a) Case III.h proposes a lower investment on PV units (see Fig. 4); b) Case III.h proposes the allocation of an additionally fixed CB at node 90; and c) Case III.h proposes different conductor type at circuit 35-36 (see Table II). The results obtained for both annual resolutions shows that a higher time-resolution may result in a more suitable plan. The higher time-resolution is, the more precise the approximation become, and consequently, a more satisfactory result can be obtained; however, it negatively affects the computational efficiency.

In summary, the proposed model provides appropriate robust solutions for cases I-III under two annual resolution conditions within a few iterations while the obtained maximum gap was less than $0.2 \%$. Table I presents the gaps, upper and lower bounds, and pollutant emissions in each iteration. To fulfill the technical, operational, and environmental conditions of the 


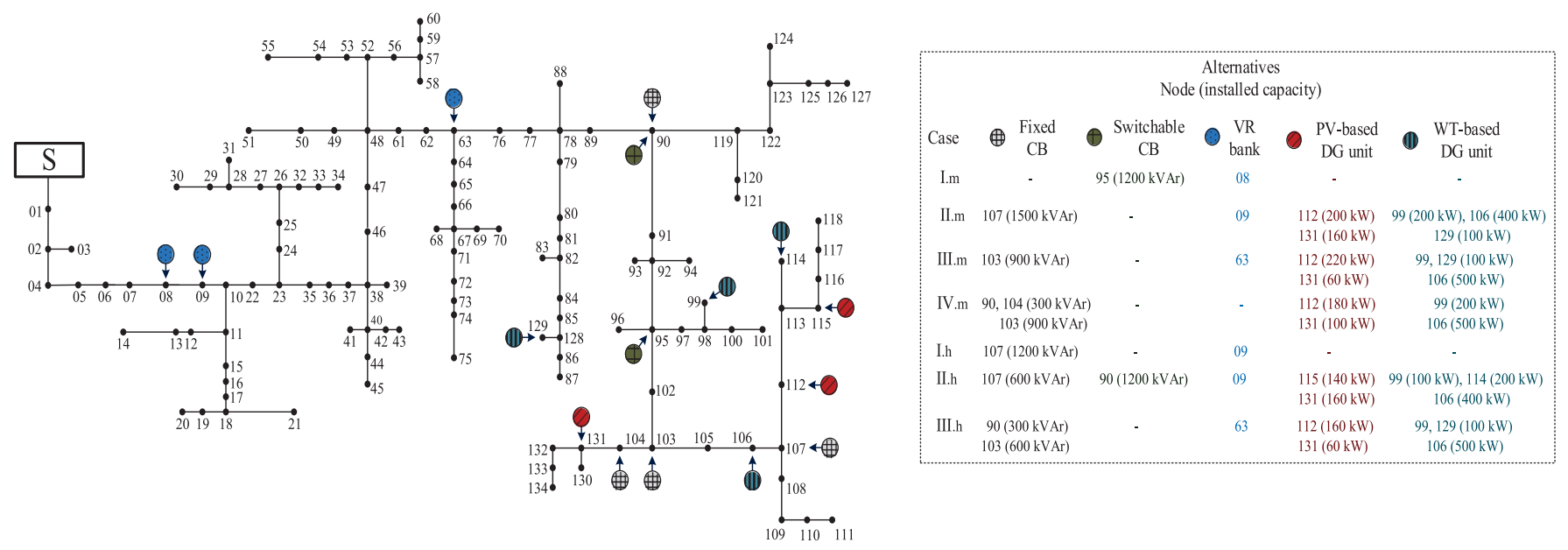

Fig. 4. 135-node distribution test system and proposed investment in renewable-based DG units, CBs, and VRs for each Case.

TABLE II

CONDUCTOR REPLACEMENT PROPOSAL For MODERATE- AND High-RESOLUTION

\begin{tabular}{l|c|c|c|c|c|c}
\hline Circuit & Initial & \multicolumn{5}{|c}{ Cases (New conductor) } \\
\cline { 3 - 6 }$(m-n)$ & Conductor & I.m & III.m & IV.m & I.h & III.h \\
\hline $01-02$ & AA4 & AA5 & AA6 & AA6 & AA5 & AA6 \\
$02-04$ & AA4 & AA6 & AA5 & AA6 & AA6 & AA5 \\
$04-05$ & AA4 & AA6 & AA6 & AA6 & AA6 & AA6 \\
$05-06$ & AA4 & AA5 & AA6 & AA6 & AA5 & AA6 \\
$06-07$ & AA4 & AA5 & AA6 & AA6 & AA5 & AA6 \\
$07-08$ & AA4 & AA6 & AA6 & AA6 & AA6 & AA6 \\
$08-09$ & AA4 & AA6 & AA6 & - & AA6 & AA6 \\
$09-10$ & AA4 & AA6 & AA6 & AA5 & AA6 & AA6 \\
$10-22$ & AA4 & AA5 & AA5 & AA5 & AA6 & AA5 \\
$22-23$ & AA4 & AA6 & AA5 & AA5 & AA6 & AA5 \\
$23-35$ & AA4 & AA5 & - & - & AA5 & - \\
$35-36$ & AA4 & AA6 & AA6 & - & AA6 & AA5 \\
$36-37$ & AA4 & AA5 & - & - & AA5 & - \\
$37-38$ & AA4 & AA5 & - & - & AA5 & - \\
$38-46$ & AA4 & AA5 & - & - & AA6 & - \\
46-47 & AA4 & AA5 & - & - & AA5 & - \\
\hline
\end{tabular}

initially-violated 135-nodes EDS, a set of compelling planning alternatives was obtained for each case study; this information is summarized in Table II and Fig. 4. Table II contains the conductor replacement of overloaded circuit with the new ones while Fig. 4 identifies the location, capacity and type of $\mathrm{CBs}$ and renewable-based DG units, and allocation of VRs for each case.

It is worth noting that, since the proposed adaptive robust formulation is based on an approximated MILP model and to obtain this model some relaxations and approximations were applied. In order to evaluate the precision of the proposed formulation, all the decision variables (i.e., location and output power of PV and WT units, location and reactive output power of CBs, location of VRs, and conductor type to each circuit of the EDS) of the solution found from the adaptive robust short-term planning approach, for each case, was used to solve a conventional power flow. Comparing the solution obtained from the MILP model with the conventional power flow, the maximum relative error percentage between the outcomes was about $0.016 \%$. Besides, the voltage magnitude relative error percentage obtained was less than $0.002 \%$, which is negligible. These results validate the acceptable precision of the proposed MILP model as a suitable approximated tool for the steady-state operation of an EDS.

\section{CONCLUSION}

In this work, unlike the approaches presented in the literature, a strategy for the short-term planning of electrical distribution systems considering multiples investment alternatives and siting and sizing of renewable-based DG units, based on a two-stage robust optimization has been proposed. The proposed planning scheme represents a hierarchical structure decision in which the investment actions are made by the master problem, while the sub-problem determines the adjustable decisions. Consequently, this robust planning framework can be a useful tool for distribution companies to address the requirements of the consumers, energy quality, reliability, and environmental criteria imposed by regulatory agencies.

Several case studies have been analyzed under moderateand high-resolution conditions to represent the seasonal characteristics and weather factors. Under The first condition, the optimal plan was obtained with a high computational efficiency, while under the second condition a similar plan obtained with a few differences and lower computational efficiency. This shows that regardless of time resolution, the proposed formulation captures an appropriate result. Therefore, the numerical results reveal the effectiveness of the proposed approach as a robust optimization tool for the decision-making process, where for each case, a set of planning actions is determined that satisfy economic-environmental criteria of a short-term planning problem while maximizing the efficiency of an initially violated EDS.

Due to the scope of this paper and for practical purposes, the average energy cost was represented for each time interval. In fact, this value depends on the electricity market price and is subject to variability, and hence, prediction errors may arise. On the other hand, the pollutant emission at distribution level was considered via a carbon cap policy. This policy is a binding constraint that affects the flexibility of the distribution system. Therefore, the optimal dispatch should appropriately change to satisfy this predefined limit. The prediction errors in energy cost and other carbon policies are the prospect of the further research work. 


\section{REFERENCES}

[1] I. Szuvovivski, T. S. P. Fernandes, and A. R. Aoki, "Simultaneous allocation of capacitors and voltage regulators at distribution networks using genetic algorithms and optimal power flow." Int. J. Elect. Power Energy Syst., vol. 40, no. 1, pp. 62-69, Sep. 2012.

[2] A. A. Abou El-Ela, R. A. El-Sehiemy, A. M. Kinawy, and M. T. Mouwafi, "Optimal capacitor placement in distribution systems for power loss reduction and voltage profile improvement," IET Gener. Trans. Dist., vol. 10, no. 5, pp. 1209-1221, Apr. 2016.

[3] V. Farahani, S. H. H. Sadeghi, H. Askarian Abyaneh, S. M. M. Agah, and K. Mazlumi, "Energy loss reduction by conductor replacement and capacitor placement in distribution systems," IEEE Trans. Power Syst., vol. 28, no. 3, pp. 2077-2085, Aug. 2013.

[4] A. Asrari, S. Lotfifard, and M. Ansari, "Reconfiguration of smart distribution systems with time varying loads using parallel computing," IEEE Trans. Smart Grid, vol. 7, no. 6, pp. 2713-2723, Nov. 2016.

[5] B. R. Pereira, A. M. Cossi, and J. R. Mantovani, "Multiobjective shortterm planning of electric power distribution systems using NSGA-II," J. Control Autom. Elect. Syst., vol. 24, no. 3, pp. 286-299, Apr. 2013.

[6] T. Adefarati and R. C. Bansal, "Integration of renewable distributed generators into the distribution system: a review," IET Renewable Power Gener., vol. 10, no. 7, pp. 873-884, Jul. 2016.

[7] M. F. Shaaban, Y. M. Atwa, and E. F. El-Saadany, "DG allocation for benefit maximization in distribution networks," IEEE Trans. Power Syst., vol. 28, no. 2, pp. 639-649, May 2013.

[8] U. Sultana, A. B. Khairuddin, M. M. Aman, A. S. Mokhtar, and N. Zareen, "A review of optimum DG placement based on minimization of power losses and voltage stability enhancement of distribution system," Renewable Sustain. Energy Rev., vol. 63, pp. 363-378, Sep. 2016.

[9] B. R. Pereira, G. R. da Costa, J. Contreras, and J. R. Mantovani, "Optimal distributed generation and reactive power allocation in electrical distribution systems," IEEE Trans. Sustain. Energy, vol. 7, no. 3, pp. 975-984, Jul. 2016.

[10] M. B. Jannat and A. S. Savić, "Optimal capacitor placement in distribution networks regarding uncertainty in active power load and distributed generation units production," IET Gener. Transmiss. Distrib., vol. 10, no. 12, pp. 3060-3067, Aug. 2016.

[11] D. Q. Rodezno, J. Wang, M. Sarker, and A. Padilla-Feltrin, "Stochastic assessment of distributed generation hosting capacity and energy efficiency in active distribution networks," IET Gener. Transmiss. Distrib., vol. 11, no. 18 , pp. $4617-4625$, Dec. 2017.

[12] M. Ahmadigorji, N. Amjady, and S. Dehghan, "A robust model for multiyear distribution network reinforcement planning based on informationgap decision theory," IEEE Trans. Power Syst., vol. 33, no. 2, pp. 13391351, Mar. 2018

[13] R. R. da Cunha Paiva, A. C. Rueda-Medina, and J. R. Mantovani, "Shortterm electrical distribution systems planning considering distributed generation and reliability," J. Control Autom. Elect. Syst., vol. 28, no. 4, pp. 552-566, May 2017.

[14] M. Pourakbari-Kasmaei, M. J. Rider, and J. R. S. Mantovani, “An unequivocal normalization-based paradigm to solve dynamic economic and emission active-reactive OPF (Optimal Power Flow)," Energy, vol. 73, pp. 554-566, Aug. 2014.

[15] M. van der Hoeven, "World Energy Outlook," Int. Energy Agency, Paris Cedex 15, France, Tech. Rep. 1, 2012.

[16] B. Zeng, J. Zhang, X. Yang, J. Wang, J. Dong, and Y. Zhang, "Integrated planning for transition to low-carbon distribution system with renewable energy generation and demand response," IEEE Trans. Power Syst., vol. 29, no. 3, pp. 1153-1165, May 2014.

[17] P. Kayal and C. K. Chanda, "Strategic approach for reinforcement of intermittent renewable energy sources and capacitor bank for sustainable electric power distribution system," Int. J. Elect. Power Energy Syst., vol. 83, pp. 335-351, Dec. 2016.

[18] S. S. Tanwar and D. K. Khatod, "Techno-economic and environmental approach for optimal placement and sizing of renewable DGs in distribution system," Energy, vol. 127, pp. 52-67, May 2017.

[19] O. D. M. Dominguez, M. Pourakbari-Kasmaei, J. R. Mantovani, and M. Lavorato, "Environmentally committed short-term planning of electrical distribution systems considering renewable based DG siting and sizing," in Proc. Int. Conf. Environ. Elect. Eng. Conf., Milan, Italy, Jul. 2017, pp. 1-6.
[20] M. Resener, S. Haffner, L. A. Pereira, and P. M. Pardalos, "Optimization techniques applied to planning of electric power distribution systems: a bibliographic survey," Energy Syst., pp. 1-37, Jan. 2018.

[21] S. Montoya, J. I. Muñoz, and J. Contreras, "A stochastic investment model for renewable generation in distribution systems," IEEE Trans. Sustain. Energy, vol. 6, no. 4, pp. 1466-1474, Oct. 2015.

[22] Y. Atwa, El-Saadany, M. Salama, and R. Seethapathy, "Optimal renewable resources mix for distribution system energy loss minimization," IEEE Trans. Power Syst., vol. 25, no. 1, pp. 360-370, Feb. 2010.

[23] I. Staffell and S. Pfenninger, "Using bias-corrected reanalysis to simulate current and future wind power output," Energy, vol. 114, pp. 1224-1239, Nov. 2016.

[24] S. Pfenninger and I. Staffell, "Long-term patterns of European PV output using 30 years of validated hourly reanalysis and satellite data," Energy, vol. 114, pp. 1251-1265, Nov. 2016

[25] U.S. Energy Information Administration, Washington, DC, USA, "Average retail price of electricity," 2017. [Online]. Available: https://www.eia. gov/electricity/data/browser/topic

[26] D. Bertsimas and M. Sim, "Robust discrete optimization and network flows," Math. Program., vol. 98, pp. 49-71, May 2003.

[27] A. Ben-Tal, A. Goryashko, E. Guslitzer, and A. Nemirovski, "Adjustable robust solutions of uncertain linear programs," Math. Program., vol. 99 no. 2, pp. 351-376, Mar. 2004

[28] B. Zeng and L. Zhao, "Solving two-stage robust optimization problems using a column-and-constraint generation method," Oper. Res. Lett., vol. 41 , pp. 457-461, Feb. 2013.

[29] S. P. Boyd and L. Vandenberghe, Convex Optimization. Cambridge, U.K. Cambridge Univ. Press, 2004

[30] U.S. Energy Information Administration, Washington, DC, USA, "State carbon dioxide emissions data," 2017. [Online]. Available: https://www. eia.gov/environment/emissions/state/

Ozy Daniel Melgar-Dominguez received the B.Sc. degree in electrical engineering from the National Autonomous University of Honduras, Tegucigalpa, Honduras, in 2011, the M.Sc. degree in electrical engineering, in 2015, from the São Paulo State University, Ilha Solteira, Brazil, where he is currently working toward the Ph.D. degree in electrical engineering.

His research interests include the development of methodologies for the optimization, integration of renewable energy systems, and planning and control of electrical power systems.

Mahdi Pourakbari-Kasmaei (S'10-M'15) received the B.Sc. and M.Sc degrees in electrical engineering, electronics and control engineering in 2005 and 2008, respectively, and the Ph.D. degree in electrical engineering, power systems, from the São Paulo State University (UNESP), Ilha Solteira, Brazil, in 2015.

From 2008 to 2011, he was a Project Executive of three practical projects and also a Consultant in an electric power distribution company where he received the exemplary engineer award. He was a Postdoctoral Fellow with UNESP. He also was a Visiting Researcher with UCLM, Ciudad Real, Spain, for about 15 months. He is currently a Researcher with Aalto University, Espoo, Finland. His research interests include power systems planning, operations and economics, smart grids, electricity market, and environmental issues.

José Roberto Sanches Mantovani (M'06) received the B.Sc. degree from the São Paulo State University (UNESP), Ilha Solteira, Brazil, in 1981, and the M.Sc. and Ph.D. degrees in electrical engineering from the University of Campinas, Campinas, Brazil, in 1987 and 1995, respectively.

$\mathrm{He}$ is currently a Professor with the Department of Electrical Engineering, UNESP. His research interests include the development of methodologies for the optimization, planning, and control of electrical power systems, and applications of artificial intelligence in power systems. 